\title{
Las redes de acción pública como sistemas asociativos complejos: Problemas y mecanismos de integración ${ }^{1}$
}

\author{
Matilde Luna y José Luis Velasco² - UNAM
}

\section{Resumen}

Este trabajo analiza las propiedades sistémicas de las interacciones en las redes de gobernanza. Se identifican cuatro propiedades básicas: la autonomía individual de los participantes, la autonomía política de la red, la interdependencia de sus componentes y el dinamismo. También se analizan cuatro mecanismos y procesos para la integración y toma de decisiones: la confianza, la traducción, la negociación y la deliberación. Se concluye que para funcionar adecuadamente, las redes deben lograr un delicado equilibrio entre estos cuatro elementos.

Palabras clave: Redes de gobernanza - Sistemas asociativos complejos - Redes de acción pública - Mecanismos de integración - Deliberación y negociación

\begin{abstract}
This paper analyzes the systemic properties of the interaction that takes place in governance networks. We identify four basic properties: individual autonomy of participants, political autonomy of the network, interdependence of its members, and dynamism. We also analyze four integrative and decision-making mechanisms and processes: trust, translation, negotiation and deliberation. Our main conclusion is that to function adequately, networks must achieve a delicate balance among these four elements.
\end{abstract}

Key words: Governance networks - Complex associative systems - Public action networks - Integrative mechanisms - Deliberation and negotiation.

En los espacios públicos es ahora común la configuración de entidades más o menos formales orientadas a afrontar problemas o conflictos de orden colectivo mediante la cooperación. Estas entidades, identificadas como "redes de políticas públicas" y más ampliamente como "redes o estructuras de gobernanza" se caracterizan por una composición de actores heterogéneos que en combinaciones variables incluyen agencias gubernamentales, organizaciones sociales, movimientos colectivos, comunidades epistémicas, asociaciones de intereses, empresarios, expertos, ciudadanos individuales y coaliciones inestables de partidos y organismos de la sociedad civil. Se trata de configuraciones que desde la perspectiva del Análisis de Redes Sociales pueden ser caracterizadas como estructuras policéntricas o multicéntricas, formadas por vínculos débiles o "puentes" y que suelen ser muy dinámicas e inestables.

\footnotetext{
1 Este trabajo incluye un resumen de Luna y Velasco (en prensa), y retoma hallazgos de otras investigaciones de los autores, tanto de carácter conceptual como empírico.

Enviar correspondencia a: (Matilde Luna Ledesma mluna@servidor.unam.mx y José Luis Velasco jose.velasco@unam.mx)

${ }^{2}$ Instituto de Investigaciones Sociales, UNAM.
} 
REDES- Revista hispana para el análisis de redes sociales

Vol.17,\#4, Diciembre 2009

http: //revista-redes.rediris.es

El propósito de este trabajo es identificar las propiedades sistémicas de las interacciones en las redes de gobernanza, así como sus características e implicaciones en el estudio de la política y el análisis social. Más que atender a la morfología de la red, el trabajo pone el acento en los procesos que hacen posible la integración de actores heterogéneos y relativamente autónomos, y que tienen lugar en las interfaces del sistema de relaciones: la creación de confianza (relativa a la cohesión de la red), la traducción (que alude a la red como un sistema de comunicación) y la toma de decisiones (que se refiere a la manera en que se procesan los conflictos y se coordinan las acciones entre actores heterogéneos: con intereses, necesidades y preferencias distintas). Sobre esta base, se retoma un tema de interés del Análisis de Redes Sociales: el relativo al desempeño de las redes.

\section{Descripción de las redes de acción pública}

Con distintos niveles de formalidad, las redes de acción pública operan por lo general en ciertos campos de la política social como la salud y la educación. Con frecuencia, atraviesan distintos dominios, involucrando la participación de diferentes agencias gubernamentales y de grupos sociales diversos, como en el caso de las políticas de innovación, las políticas ambientales, o las políticas de capacitación. En otros casos, las redes se orientan a la solución de problemas complejos relacionados con la inseguridad, el desempleo, la pobreza o la inequidad de género. En función de sus alcances territoriales presentan también combinaciones variables. Por ejemplo, particularmente en periodos de crisis, resultan en arreglos institucionales de alcance nacional pero con una participación directa de organismos internacionales, gubernamentales y no gubernamentales, o pueden derivar en arreglos institucionales de alcance multinacional.

De hecho, un elemento que distingue la particularidad del espacio público hoy es la estructuración de relaciones complejas que, en la búsqueda de soluciones a problemas comunes, involucran la comunicación e interacción entre individuos y colectividades con diferentes lógicas y lenguajes. En sentido estricto, estas estructuras no son políticas (es decir, guiadas por la búsqueda del poder y regidas por la ley y la coerción), económicas (orientadas a la obtención de ganancias y regidas por la competencia) o sociales (gobernadas estrictamente por normas sociales).

Las estructuras de este tipo se crean para atender problemas o áreas de política que dada su complejidad y/o nivel de conflictividad no pueden ser atendidos por los 
REDES- Revista hispana para el análisis de redes sociales

Vol.17,\#4, Diciembre 2009

http: //revista-redes.rediris.es

canales institucionalizados tradicionales, y por lo tanto su propósito fundamental es contribuir al orden social mediante la toma de decisiones o acuerdos de carácter colectivo. Estas organizaciones suelen ser promovidas, dotadas de personal, financiadas, o supervisadas por las propias autoridades legales y legítimamente reconocidas, y cuentan por lo general con la participación de expertos o especialistas en la materia. Es por ello que con frecuencia la literatura se refiere a los miembros como "participantes" que tienen un interés en el problema en cuestión (stake-holders) y los que tienen conocimiento sobre el problema (knowledge-holders), para distinguirlos a los representantes en el sentido estricto del término (right-holders).

Buena parte de estos sistemas asociativos complejos (SACs) son organizaciones de organizaciones, o tienen una composición mixta de organizaciones e individuos, quienes por lo general participan como "representantes" de otras organizaciones, sectores o grupos involucrados en la atención de un tema o un problema específico. Es el caso también de asociaciones individuales en las que diversos interesados como los beneficiarios, los donantes o en general los organismos financieros y patrocinadores, o los expertos, tienen una influencia importante.

La expresión más clara de estas organizaciones complejas son aquellos consejos y comisiones que cuentan con la participación de agentes políticos, empresariales y de la sociedad civil que tienen una importante influencia política o incluso un poder de veto en asuntos de interés público, y que con frecuencia cuentan con la participación de empresas y organismos gubernamentales y no gubernamentales de carácter supranacional. Se trata de sistemas asociativos complejos que se han generalizado como resultado de procesos simultáneos de diferenciación e interdependencia entre diferentes subsistemas: el Estado, el mercado y la sociedad civil (Messner 1999), pero también como consecuencia de procesos de globalización y de comunicación que han impactado la forma en que se integran las organizaciones de acción colectiva. Las redes de políticas cumplen distintas funciones, entre ellas, información y asistencia, consulta, implementación, diseño de políticas, fiscalización y regulación.

En este tipo de redes asociativas, los participantes se encuentran insertos en diferentes ámbitos institucionales: el mercado, las instituciones políticas o la sociedad civil; persiguen propósitos funcionales diferentes: la ganancia, el poder o causas diversas, y sus respectivos recursos son con frecuencia inconmensurables, como puede ser el caso de los recursos financieros, de poder o de movilización 
REDES- Revista hispana para el análisis de redes sociales

Vol.17,\#4, Diciembre 2009

http://revista-redes.rediris.es

política, respectivamente. Por esta razón presentan lo que March (1997) llama "inconsistencias interactivas".

De manera meramente ilustrativa pueden mencionarse los siguientes casos de SACs, los que han tenido diferentes niveles de éxito en la consecución de sus propósitos:

En la Unión Europea, la Comisión Europea ha promovido la creación de redes como parte de sus políticas, ya sea en la búsqueda de objetivos comunes, en el establecimiento de prioridades, en la implementación de programas o en la definición conjunta de objetivos, métodos y estrategias. Como principio, se reconoce que las redes de políticas son suficientemente flexibles para procesar la gran diversidad de culturas administrativas y de estructuras de los países y sectores involucrados (Comisión Europea: De Bruïne y Clarotti 2001: 1).

De una índole muy diferente es el caso del llamado "Diálogo Argentino", que dio lugar a una estructura creada como salida a la crisis institucional de los primeros años de la década de 2000, convocada por el gobierno, la iglesia y el Programa de las Naciones Unidas para el Desarrollo, y donde participaron alrededor de 300 entidades, incluyendo partidos políticos, ONGs, empresas y grupos emergentes como asociaciones de ahorradores y clubes de trueque, entre otros.

En México, puede mencionarse el caso del "Acuerdo de Chapultepec". Bajo el liderazgo de Carlos Slim (uno de los empresarios más ricos del mundo), el Acuerdo Nacional para la Unidad, el Estado de Derecho, el Desarrollo, la Inversión y el Empleo, fue firmado en septiembre de 2005, en la coyuntura de la sucesión presidencial, que como el propio Slim señaló públicamente entonces, se anunciaba muy competida. Presentado como un acuerdo de la sociedad civil, éste contó con el aval inicial de alrededor de 500 personas, entre ellas dirigentes de importantes organizaciones empresariales, del sector laboral, de asociaciones profesionales y de la sociedad civil, así como personalidades del medio artístico y del deporte. A este acuerdo se sumaron los actores políticos; entre ellos, lo suscribieron miembros del gabinete, dirigentes de los partidos políticos, candidatos presidenciales y la Conferencia Nacional de Gobernadores, integrada por el conjunto de los gobernadores procedentes de los distintos partidos políticos. El máximo órgano de coordinación del "Acuerdo de Chapultepec", la Comisión de Información y Seguimiento, integrada por 20 personas y de carácter colegiado, es ilustrativo de la heterogeneidad de un sistema asociativo, como puede observarse en el siguiente cuadro relativo a su composición. 
REDES- Revista hispana para el análisis de redes sociales

Vol.17,\#4, Diciembre 2009

http: //revista-redes.rediris.es

\begin{tabular}{|c|c|}
\hline Sectores participantes & I dentidad ${ }^{3}$ \\
\hline Organizaciones empresariales & $\begin{array}{l}\text { - Presidente del Consejo Coordinador Empresarial } \\
\text { - } \quad \text { Presidente del Consejo Mexicano de Hombres de } \\
\text { Negocios } \\
\text { - Representante del Consejo Nacional Agropecuario }\end{array}$ \\
\hline Organizaciones del sector laboral & $\begin{array}{l}\text { - } \quad \text { Presidente del Congreso del Trabajo } \\
\text { - } \quad \text { Presidente de la Confederación de Trabajadores de } \\
\text { México } \\
\text { - } \quad \text { Presidente de la Unión Nacional de Trabajadores }\end{array}$ \\
\hline $\begin{array}{l}\text { Organizaciones del sector } \\
\text { campesino }\end{array}$ & - $\quad$ Presidente de la Confederación Nacional Campesina. \\
\hline Grupos económicos & $\begin{array}{ll}\text { - } & \text { Carso } \\
\text { - } & \text { Telmex } \\
\text { - } & \text { Comex } \\
\text { - } & \text { Cemex } \\
\text { - } & \text { Corporación Durango }\end{array}$ \\
\hline Organizaciones de la Sociedad Civil & $\begin{array}{l}\text { - Presidente de México Unido contra la Delincuencia } \\
\text { - Presidente del Fondo Mexicano para la Educación y el } \\
\text { Desarrollo }\end{array}$ \\
\hline Medios de comunicación & 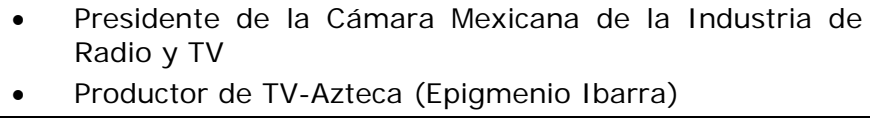 \\
\hline Ciencia & Premio Nobel (Mario Molina) \\
\hline Academia & $\begin{array}{l}\text { - Miembro de la Junta de Gobierno de la Universidad } \\
\text { Nacional Autónoma de México (Rolando Cordera) }\end{array}$ \\
\hline Medio cultural & Ängeles Mastretta (escritora) \\
\hline Medio artístico & Emmanuel Acha Martínez (“Emmanuel”, cantante) \\
\hline
\end{tabular}

Cuadro 1. Composición de la Comisión de Información y Seguimiento del Acuerdo de Chapultepec.

Ya sea que se trate de organizaciones multisectoriales o especializadas, estas estructuras complejas comparten un conjunto de propiedades, y en mayor o menor grado comparten también un conjunto de características particulares. También por sus rasgos y naturaleza, este tipo de estructuras plantean importantes problemas de integración para coordinar las acciones y procesar los conflictos potenciales derivados de una composición heterogénea.

\section{Las redes como estructuras o sistemas complejos}

La noción de las redes como estructuras o sistemas complejos no es ajena al Análisis de Redes Sociales, aunque en general se ha privilegiado el estudio de las redes densas. En la búsqueda por establecer conexiones entre las interacciones individuales en el nivel micro, y los grandes patrones de relaciones sociales, a principios de los años setenta Granovetter (1973) plantearía el argumento de "la fortaleza de las ligas débiles", en donde la fortaleza es informativa y la debilidad se

\footnotetext{
${ }^{3}$ Cabe señalar, que varios de los "representantes" tienen afiliaciones institucionales múltiples, ya que transitan entre las instituciones políticas, las estructuras del mercado y las organizaciones de la sociedad civil.
} 
REDES- Revista hispana para el análisis de redes sociales

Vol.17,\#4, Diciembre 2009

http: //revista-redes.rediris.es

refiere a la intensidad de los vínculos interpersonales, entendida "como una combinación lineal de tiempo, intensidad emocional, intimidad y servicios recíprocos" (p. 1361). El vínculo débil es un "puente", es decir, la única línea en una red que comunica dos puntos, que conecta entre sí diferentes grupos, cada uno formado por individuos con vínculos fuertes (intensos). Varios desarrollos recientes en esta línea incluyen las contribuciones de Burt (1992) sobre los hoyos estructurales, y de Valente (1995) sobre la estructura radial.

Burt desarrollaría el argumento del hoyo estructural con base en la noción de red como una entidad compleja. El autor enfatiza la importancia de la autonomía de las redes y de sus componentes; de las redes abiertas (no redundantes), más que de las cerradas, y de la acción estratégica o selectiva de los actores. Desde una perspectiva económica, Burt plantea que los "hoyos estructurales" o regiones dispersas que existen entre regiones densas de relaciones, representan oportunidades para negociar flujos de información original (o nuevas ideas) entre individuos (o empresas) que se encuentran en los lados opuestos de un hoyo en la estructura social, lo que a su vez repercute en altos niveles de retorno económico (Burt 1992; Walker, Kogut y Shan, 2000). Valente, por su parte, en su estudio sobre la difusión de las innovaciones intenta operacionalizar las ligas débiles como la presencia de individuos que vinculan a grupos de otra manera desconectados, a quienes identifica como individuos con redes personales radiales, que define por oposición a las redes individuales densas o con un alto grado de interconexión. Valente concluye que la importancia de los individuos ligas-débiles no es equivalente a su autoridad. Sin embargo, reconoce que la "radialidad" es una medida pobre del concepto de liga débil. Aquí observamos el problema desde la perspectiva de los procesos y mecanismos de integración de actores heterogéneos e interdependientes.

\section{Propiedades de los sistemas asociativos complejos (SACs)}

Las redes pueden ser tipificadas de acuerdo con diferentes criterios, según los propósitos que se buscan. Un criterio pertinente es el de su nivel de complejidad, que puede definirse en los siguientes términos, relativos a las propiedades sistémicas de sus interacciones:

Por el nivel de autonomía individual de los participantes, esto es, por la capacidad de elección de los individuos o "representantes". 
REDES- Revista hispana para el análisis de redes sociales

Vol.17,\#4, Diciembre 2009

http://revista-redes.rediris.es

Por el nivel de autonomía política de la red, es decir, la capacidad de autorregulación del sistema asociativo. ${ }^{4}$

Por el nivel de interdependencia entre sus unidades (individuos, colectividades u organizaciones)

Por el nivel de dinamismo de la red, relacionado con su inestabilidad y potencial para generar nuevas redes. ${ }^{5}$

Desde esta perspectiva puede decirse que las redes de gobernanza se distinguen por un nivel de complejidad que va de medio a alto. Esto las distingue claramente de los sistemas asociativos menos complejos, como serían las cámaras empresariales o las asociaciones gremiales (ver cuadro 2).

\begin{tabular}{|c|c|c|}
\hline $\begin{array}{l}\text { Propiedades de las } \\
\text { interacciones }\end{array}$ & Altamente complejos & De baja complejidad \\
\hline Autonomía individual & $\begin{array}{l}\text { - Nivel razonable de independencia } \\
\text { para decidir }\end{array}$ & $\begin{array}{l}\text { - Mayor nivel de cohesión social } \\
\text { y espíritu de cuerpo }\end{array}$ \\
\hline Autonomía política & $\begin{array}{l}\text { - Significativa capacidad de } \\
\text { autorregulación } \\
\text { - Reglas internamente consensuadas } \\
\text { y flexibles }\end{array}$ & $\begin{array}{l}\text { - Regulados por una autoridad } \\
\text { externa } \\
\text { - Reglas de interacción fijas y } \\
\text { preestablecidas }\end{array}$ \\
\hline I nterdependencia & $\begin{array}{l}\text { - Dependencia mutua de recursos } \\
\text { - Cada participante tiene una cierta } \\
\text { autonomía y ninguno tiene } \\
\text { autoridad absoluta }\end{array}$ & $\begin{array}{l}\text { - La asociación controla sus } \\
\text { propios recursos } \\
\text { - Subordinación del individuo a } \\
\text { los fines de la asociación }\end{array}$ \\
\hline Dinamismo & $\begin{array}{l}\text { - Fronteras organizativas difusas } \\
\text { - Capacidad significativa de creación } \\
\text { de nuevos sistemas asociativos: } \\
\text { fusiones, separaciones } \\
\text { - Niveles significativos de elasticidad } \\
\text { de la membresía, objetivos, } \\
\text { recursos }\end{array}$ & $\begin{array}{l}\text { - Fronteras organizativas bien } \\
\text { definidas } \\
\text { - Primacía de la supervivencia y } \\
\text { fortalecimiento de la propia } \\
\text { asociación } \\
\text { - Estructuras y propósitos } \\
\text { duraderos }\end{array}$ \\
\hline
\end{tabular}

Cuadro 2. Tipología de sistemas asociativos o asociaciones por niveles de complejidad.

Estas propiedades, traducidas al campo de la morfología de las redes (o el ARS), se manifiestan en una configuración policéntrica o multicéntrica, y en un sistema de relaciones de ligas débiles, que es resultado de una membresía heterogénea y elástica, donde los actores se orientan con base en diferentes principios de integración, tales como el intercambio o la ganancia (relativo al sistema económico), la autoridad legítima o la ley (referente al sistema político), y la solidaridad (propia de la comunidad), que no siempre son consistentes entre sí. En las redes públicas, puede decirse, opera el principio de la fortaleza de las ligas

\footnotetext{
${ }^{4}$ Sobre esta noción de doble autonomía con referencia a la asociatividad y la democracia véase Warren (2001), y para el caso de las redes de conocimiento Luna y Velasco (2006).

${ }^{5}$ Schneider, et al (2006) identifican a los sistemas asociativos complejos como aquellos con un alto grado de dinamismo que se expresa en el establecimiento de nuevas asociaciones, en fusiones o en separaciones.
} 
REDES- Revista hispana para el análisis de redes sociales

Vol.17,\#4, Diciembre 2009

http://revista-redes.rediris.es

débiles, donde la debilidad se refiere al bajo nivel de proximidad entre los actores y la fortaleza es informativa.

Los sistemas complejos son autónomos en un doble sentido. Por una parte, cada uno de sus miembros (individuales o colectivos) es autónomo en cierta medida y permanece como tal aun cuando la interacción y la colaboración se intensifiquen. Por otra parte, la red en su conjunto es autónoma, en el sentido de que no está sujeta directamente a una entidad superior que regule sus acciones. Esta doble autonomía significa que no hay reglas preestablecidas que determinen rígidamente los derechos y obligaciones de los miembros y los procedimientos que sus interacciones deben seguir (auto-regulación), sino que estas reglas son flexibles e internamente consensuadas, y que los participantes son razonablemente libres para expresar sus opiniones y elegir sus opciones. El hecho de que ningún miembro tengan una total autoridad y todos tengan una cierta autonomía se manifiesta en una localización dinámica del liderazgo en la estructura de la red. Por la misma razón, estas redes operan en el contexto de altos niveles de incertidumbre respecto a sus procedimientos, pero también con relación a sus resultados, ya que las reglas de interacción, la delimitación de los objetivos, la definición de los problemas y la manera de resolverlos, se construyen colectiva y autónomamente por los participantes. Para construir y alcanzar metas comunes, las redes dependen de la comunicación y el flujo de información, e implican una tensión entre la cooperación y el conflicto derivados de intereses, recursos y necesidades diversas.

Cabe señalar que la autonomía individual y política que distingue a estos sistemas no es una propiedad fija, sino que se define y redefine en el proceso de la interacción e implica una permanente presión de "colonización" por el Estado (los partidos, los legisladores, los reguladores, el gobierno), el mercado u otros organismos de la sociedad civil, o por los patrocinadores o los expertos. Sin embargo, las presiones pueden ser atenuadas mediante normas, mecanismos institucionales y reglas operativas que permiten procesar y resolver las inconsistencias y conflictos. Por ejemplo, las normas relativas a la composición y representación equitativa de sus miembros, los mecanismos de rotación de la dirigencia, el predominio de una representación de corte liberal y no impositivo, y los propios mecanismos de decisión, pueden favorece la autonomía u obstaculizarla.

En cuanto a la interdependencia, básicamente se finca en la dependencia mutua de los recursos de los participantes para llegar a una solución. Schmitter (2001) describe bien la situación de interdependencia en el proceso de la decisión cuando 
REDES- Revista hispana para el análisis de redes sociales

Vol.17,\#4, Diciembre 2009

http://revista-redes.rediris.es

afirma que el problema de la formación de consensos remite a formas horizontales de interacción entre actores que tienen objetivos conflictivos, pero que son suficientemente independientes entre sí como para que ninguno pueda imponer una solución a otro, y suficientemente interdependientes como para que todos los involucrados pierdan si no se encuentra una solución. Concluye que las organizaciones participantes no tienen que ser iguales en riqueza, tamaño o capacidades, pero deben ser capaces de apoyarse o hacerse daño mutuamente ( $p$. 7). March (1997:24), basado en Matsuyama (1995), propone tres tipos de interacción que llevan a las organizaciones a redefinir mutuamente sus decisiones y sus procesos de decisión: la competencia, la cooperación y la imitación. Schneider, et al (2006) definen un rango más amplio de categorías para hablar de las conexiones en los sistemas asociativos complejos en el ámbito de las asociaciones empresariales, con base en diferentes grados y formas de cooperación y competencia, que incluyen también la neutralidad y la ausencia de relaciones.

\section{Caracterización de los SACs}

Como consecuencia o expresión de estas propiedades, la composición de las redes es significativamente heterogénea y las interacciones entre los participantes son potencialmente conflictivas; las metas suelen ser inconsistentes y cambiantes; la autoridad y por la tanto la responsabilidad de las decisiones son con frecuencia difusas; tienen problemas de legitimidad derivados en parte de una representación imprecisa y en ocasiones ambigua; las decisiones estratégicas (que implican riesgos e incertidumbre) pueden ocurrir en cualquier punto de la estructura organizativa y en cualquier momento de la vida de la red; la confianza interpersonal y el prestigio suelen tener un papel clave como medio de observancia y compromiso entre actores con intereses, preferencias e identidades inconsistentes $y$, finalmente, en cuanto a los mecanismos de decisión, presentan una marcada preferencia por las decisiones por consenso, ya sea a través de la negociación o la deliberación.

El cuadro 3 sintetiza las principales características de las redes, contrastándolas con las de sistemas asociativos menos complejos. El nivel de heterogeneidad $u$ homogeneidad de la membresía de la red es un criterio cada vez mejor reconocido para entender la dinámica de las redes. Es importante destacar, sin embargo, que este criterio tiene significados variables. La heterogeneidad ha sido relacionada, por ejemplo, con los perfiles sociodemográficos de la membresía o los participantes (edad, sexo, nivel educativo, ocupación, etc.) o con la "distancia psicológica" o social, o simplemente con diferencias que son significativas respecto a la situación. 
REDES- Revista hispana para el análisis de redes sociales

Vol.17,\#4, Diciembre 2009

http://revista-redes.rediris.es

Sin embargo, en un nivel más básico es importante mencionar los problemas derivados de las inconsistencias en las preferencias, las identidades individuales y las afiliaciones múltiples de los participantes, que pueden generar confusión; la diversidad de intereses y recursos (información, recursos políticos, económicos y simbólicos, etc.) que afecta el intercambio; la diversidad de concepciones sobre los fines, propósitos y metas de la organización, y las diferentes concepciones sobre el problema que se aborda y las estrategias para resolverlo.

\begin{tabular}{|c|c|c|}
\hline Características & Altamente complejas & Baja complejidad \\
\hline Membresía & $\begin{array}{l}\text { - Participantes heterogéneos, con } \\
\text { niveles significativos de } \\
\text { diferenciación } \\
\text { - Criterios flexibles de membresía }\end{array}$ & $\begin{array}{l}\text { - Participantes homogéneos con } \\
\text { respecto a las características } \\
\text { relevantes de la asociación } \\
\text { - Criterios homogéneos e } \\
\text { inequívocos de membresía }\end{array}$ \\
\hline Objetivos & $\begin{array}{l}\text { - Propósitos y objetivos diversos y } \\
\text { potencialmente conflictivos } \\
\text { - Metas inconsistentes } \\
\text { - Definición y redefinición consensuada } \\
\text { de objetivos y metas }\end{array}$ & $\begin{array}{l}\text { - Propósito funcional claro } \\
\text { - Metas claras y consistentes } \\
\text { - Objetivos preestablecidos y } \\
\text { estables }\end{array}$ \\
\hline Autoridad & $\begin{array}{l}\text { - Distribución dinámica de la autoridad } \\
\text { - Importancia del prestigio y la } \\
\text { confianza en la conformación de la } \\
\text { autoridad } \\
\text { - Importancia de las interfaces o } \\
\text { espacios de frontera entre } \\
\text { subsistemas, organizaciones o } \\
\text { colectividades informales }\end{array}$ & $\begin{array}{l}\text { - Autoridad legal o legítimamente } \\
\text { instituida } \\
\text { - Importancia de la posición en la } \\
\text { estructura } \\
\text { - Importancia de las posiciones } \\
\text { ubicadas en la cúspide o el centro }\end{array}$ \\
\hline Representación & $\begin{array}{l}\text { - Concepción liberal de la } \\
\text { representación (independencia de } \\
\text { criterio del representante) } \\
\text { - Representación imprecisa y ambigua } \\
\text { - Importancia de la representación de } \\
\text { expertos e interesados }\end{array}$ & $\begin{array}{l}\text { - Representación imperativa o por } \\
\text { mandato } \\
\text { - Representación de categorías o } \\
\text { grupos bien delimitados } \\
\text { - Representación por derecho }\end{array}$ \\
\hline $\begin{array}{l}\text { Toma de } \\
\text { decisiones }\end{array}$ & $\begin{array}{l}\text { - Decisiones por consenso } \\
\text { (negociación y deliberación) }\end{array}$ & $\begin{array}{l}\text { - Decisiones personales, } \\
\text { burocráticas, por mayoría o por } \\
\text { negociación }\end{array}$ \\
\hline
\end{tabular}

Cuadro 3. Características de los sistemas asociativos por niveles de complejidad.

No cualquier relación entre actores heterogéneos puede ser catalogada como compleja. Las excepciones más evidentes son, por diversas razones, las relaciones clientelares y los arreglos corporatistas, que tienen un carácter jerárquico, y las redes encubiertas (como las redes de narcotráfico y las de terroristas), las cuales incluyen actores interdependientes pero no autónomos ${ }^{6}$. Tampoco la

\footnotetext{
${ }^{6}$ En un trabajo que recoge una variedad de estudios de caso sobre el comercio de armas y diamantes, las redes de narcotráfico y la red terrorista Al Qaeda, Brinton y Raab (2002) realizan un análisis comparativo entre lo que denominan como "redes abiertas" y "redes encubiertas". En este estudio, los autores encuentran varias similitudes pero también diferencias importantes entre cada tipo de configuración reticular; entre las diferencias son de mencionarse las siguientes: En cuanto a los mecanismos de coordinación y control, mientras que las redes abiertas se basan en el intercambio de información, las encubiertas incluyen, además del intercambio de información, la fuerza física. Respecto a la toma de decisiones, las redes abiertas son multilaterales, en tanto que las encubiertas tienden a ser
} 
REDES- Revista hispana para el análisis de redes sociales

Vol.17,\#4, Diciembre 2009

http://revista-redes.rediris.es

heterogeneidad se encuentra necesariamente asociada con el conflicto, en tanto que la homogeneidad puede dar lugar a una competencia depredadora y la heterogeneidad a una relación de complementariedad y cooperación. Desde nuestra perspectiva, la heterogeneidad es más cercana a un concepto de diferenciación institucional o funcional, que requiere por tanto de un mecanismo de traducción, que permita la construcción de un lenguaje común y posibilite el consenso. Esta heterogeneidad está asociada a la existencia de criterios flexibles e incluso ambiguos para seleccionar a los miembros y participantes.

Pero la doble relación de autonomía e interdependencia entre individuos, grupos, instituciones o subsistemas, que caracteriza a las organizaciones complejas, tiene implicaciones directas de diverso orden en el campo de la política:

En el plano de la autoridad para tomar decisiones, la doble autonomía significa que ninguno de los participantes tiene una autoridad absoluta, que cada uno de ellos tiene cierta autonomía, y que la autoridad se encuentra dinámicamente dispuesta en el sistema ${ }^{7}$. Esta situación es una fuente potencial de conflicto, en tanto que cada grupo, comunidad u organización participante tiene sus propios líderes que pueden competir por la autoridad. Más aún, esta situación crea incertidumbre, y en particular puede diluir la responsabilidad de las decisiones. Por otra parte, en los sistemas asociativos complejos las posiciones formales de autoridad no siempre corresponden a las de liderazgo. El prestigio y la confianza juegan un papel muy importante en la construcción (o destrucción) de la autoridad y el liderazgo. Diversos estudios han sugerido que el liderazgo suele estar ubicado en las interfaces del sistema, en donde la reputación o el prestigio se finca en la capacidad de los individuos para articular diferentes concepciones, intereses y lenguajes (ya sea que se trate de los "traductores", los negociadores o brokers, de los "analistas simbólicos", de los "celadores" y, en general, el "personal de frontera"8).

El problema de la legitimidad de las decisiones y sus procesos se complica porque, a diferencia de la representación política, en las organizaciones complejas no hay

unilaterales. Y finalmente, con relación al estatus de los actores, mientras que las redes abiertas involucran actores autónomos pero interdependientes, las encubiertas incluyen actores interdependientes pero no autónomos.

${ }^{7}$ Este tipo de patrón de autoridad se ha encontrado en redes complejas de empresas (Hage and Alter, 1997), redes de políticas (Messner, 1999), redes de políticas en los llamados sistemas multi-nivel como en el caso de la Unión Europea (Hoogh and Marks, 2001), y los encontramos también en las redes de conocimiento (Luna y Velasco 2006).

8 Para un resumen de la literatura sobre este punto y un estudio empírico al respecto, véase Luna y Velasco (2003). 
REDES- Revista hispana para el análisis de redes sociales

Vol.17,\#4, Diciembre 2009

http://revista-redes.rediris.es

por lo general criterios claros de representación. No solamente no es claro quién debe participar y a nombre de quién, tampoco es claro bajo qué procedimientos deben ser elegidos los participantes en la decisión. La participación de representantes de los expertos e interesados que actúan en el campo de interés de la red es crucial. Además, las afiliaciones institucionales múltiples de los participantes suelen oscurecer la transparencia de los intereses en juego ${ }^{9}$.

Los procesos de toma de decisión de los SACs se caracterizan por la búsqueda del consenso. Por lo tanto, involucran decisiones colegiadas y complicados procesos de negociación y deliberación. El consenso facilita que la organización retenga a sus miembros, y a los miembros les permite preservar su propia autonomía e identidad (Ahrne y Brunsson 2005: 442). El consenso, basado en interacciones horizontales, implica que ninguna decisión puede ser tomada en contra la oposición expresa de algún participante, y los principales dispositivos son: la deliberación (tratar de convencer a los adversarios de la buena justificación de la propia posición), el compromiso (aceptar una posición intermedia entre las preferencias expresas de los actores) y el ajuste (sopesar la intensidad de las preferencias del actor) ${ }^{10}$.

Finalmente, cabe señalar que la confianza, en sus diversas dimensiones: personal, técnica y basada en el prestigio, es -como la deliberación y la negociación- un mecanismo clave de integración de los sistemas asociativos complejos, y en particular un medio de observancia y compromiso entre miembros con objetivos e intereses inconsistentes. Como lo señalan Ahrne y Brunsson (2005) y como ha sido ampliamente reconocido en el Libro Blanco de la Unión Europea, estos sistemas no están regidos por la coerción o la ley, como en el caso del Estado, por lo que las decisiones son con frecuencia formuladas como estándares y no como reglas vinculantes. En este mismo sentido, señala March (1997), los acuerdos rara vez pueden estar especificados con precisión. "No es un mundo de contratos precisos sino de entendimientos vagos e informales y expectativas. Como consecuencia, la toma de decisiones enfatiza la confianza y la lealtad, paralelamente a una creencia ampliamente difundida de que estas cualidades son difíciles de encontrar y mantener, y de que el poder emana de ser visto como confiable". Incluso las modernas teorías de juegos, al analizar la toma de decisiones en las que participan múltiples actores, han colocado a la confianza y la reputación (más que la inteligencia en la negociación) en una posición central (26-27).

\footnotetext{
${ }^{9}$ Sobre los problemas de representación de las redes de políticas y las estructuras de gobernanza, véase Luna (2005 y 2008 , respectivamente).

${ }^{10}$ Sobre esta noción de consenso véase Schmitter 2001: 7.
} 
REDES- Revista hispana para el análisis de redes sociales

Vol.17,\#4, Diciembre 2009

http://revista-redes.rediris.es

De manera más general, puede advertirse que -por su naturaleza- los SACs involucran importantes problemas de coordinación e integración, que la evaluación de su desempeño debe considerar. En particular, la importancia de la creación de consensos pone en el centro el criterio de estabilización de un movimiento hacia la convergencia como criterio de desempeño, más que el cumplimiento de metas predeterminadas o la mera sobre-vivencia de la red. Desde esta perspectiva es pertinente preguntarse ¿cuál es la capacidad del sistema para coordinar las acciones, procesar los conflictos y eventualmente resolver los problemas que se atienden? ¿En qué medida o sentido se estabiliza la red en función de una trayectoria de convergencia?

\section{Los mecanismos de integración y sus efectos potenciales en el desempeño de las redes}

Para responder estas preguntas nos enfocamos en cuatro mecanismos de coordinación e integración que desde nuestro punto de vista son propios de los SACs: la confianza, la traducción, la negociación y la deliberación. Los dos primeros se relacionan directamente con elementos no decisionales, relativos a la cohesión y a la comunicación, respectivamente, mientras que los dos últimos se refieren directamente al proceso de decisión. El planteamiento central es que estos cuatro mecanismos operan también en otras estructuras sociales, como los mercados, la política y las comunidades. Pero lo distintivo de los SACs es que requieren la operación combinada de los cuatro y le asignan un papel especial a tres de ellos: la confianza compleja, la traducción y la deliberación.

La confianza y la cohesión ${ }^{11}$. La confianza interpersonal que surge en el contexto de relaciones sociales complejas facilita la comunicación y la cooperación entre personas, grupos y organizaciones heterogéneos. Hay una relación de confianza cuando el éxito de las acciones de una persona depende de la cooperación de alguien más; por lo tanto, implica al menos una ignorancia acerca de la conducta de los otros y la expectativa de que éstos no tratarán de obtener un provecho ilegítimo de la persona que está dispuesta a cooperar (Lane, 1998: 3; Sable, 1993). Para los SACs, tres tipos de confianza son particularmente importantes: la confianza calculada o estratégica, la técnica o basada en el prestigio y la normativa. La confianza basada en el prestigio depende sobre todo de las percepciones acerca de las capacidades y competencias de los participantes (que pueden ser individuos

\footnotetext{
11 Para una discusión más amplia de la confianza y un reporte de una investigación empírica de relaciones de colaboración entre la academia y las empresas con niveles medios y altos de complejidad, véase Luna y Velasco (2005)
} 
REDES- Revista hispana para el análisis de redes sociales

Vol.17,\#4, Diciembre 2009

http: //revista-redes.rediris.es

u organizaciones). La confianza calculada o estratégica surge de estimaciones de costos y beneficios; depende, por lo tanto, de la expectativa de beneficios mutuos que puede generar la relación. Finalmente, la confianza normativa depende de creencias y valores compartidos; se basa en la solidaridad social, más que en los beneficios esperados de la interacción.

La existencia de un tipo de confianza puede incrementar las oportunidades para el desarrollo de otros tipos; inversamente, el predominio de un tipo puede socavar la existencia de los otros. Por ejemplo, cuando la confianza se basa en expectativas puramente técnicas o estratégicas, la comunicación entre los participantes puede volverse difícil. El predominio de estas dos formas de confianza puede llegar a impedir el desarrollo de la confianza personal o normativa. Por otra parte, las relaciones de colaboración que se basan exclusivamente en la confianza normativa pueden tener mejor comunicación interpersonal pero esto puede poner en riesgo la capacidad para producir soluciones técnicamente adecuadas o estratégicamente eficientes.

Una relación inicialmente basada en la confianza normativa puede dar origen a una confianza técnica; una que esté motivada solamente por las expectativas de beneficios mutuos puede impedir el desarrollo de la confianza personal o normativa, que es la base más firme de la comunicación interpersonal. En el primer caso, la confianza puede operar como un factor para estabilizar o integrar el sistema, e incluso como un factor multiplicador para la creación de nuevas relaciones a partir del sistema de vínculos original. En el segundo caso, la confianza puede crear problemas de coordinación, eficacia o eficiencia.

Por lo tanto, un sistema asociativo complejo necesita una cantidad importante de cada uno de los tres tipos de confianza. Por esta razón es preferible no referirse a las "confianzas" normativa, estratégica y técnica como tipos separados sino como dimensiones de la confianza interpersonal compleja. Así, en este tipo de interacciones la confianza total es una combinación de las tres dimensiones. Esto explica por qué la confianza se puede generalizar entre personas con preferencias e identidades inconsistentes, con recursos e intereses distintos o con culturas organizativas diversas. ${ }^{12}$

\footnotetext{
12 En un análisis previo (Luna y Velasco 2005), encontramos que en los sistemas de colaboración complejos: (a) hay una consistencia notable entre las tres dimensiones de la confianza, (b) un tipo de confianza puede ser causa o efecto de las otras dos, lo cual significa que las cadenas transitivas de confianza (A confía en B, B confía en C, y por lo tanto A confía en C) se pueden originar en diferentes fuentes, (c) las relaciones entre estas dimensiones o componentes de la confianza pueden apoyarse mutuamente, sobreponerse o entrar en conflicto, por lo que (d) la forma en que se combinen influye sobre el origen, desarrollo y la disolución de las relaciones de colaboración.
} 
REDES- Revista hispana para el análisis de redes sociales

Vol.17,\#4, Diciembre 2009

http://revista-redes.rediris.es

En una perspectiva más amplia, se puede decir que, como una característica de los sistemas asociativos complejos $-\mathrm{y}$ como un mecanismo para integrar diferentes lógicas y lenguajes-, la confianza adquiere un carácter complejo. Es decir, necesariamente contiene cálculos, solidaridad y una percepción del prestigio de los asociados. Así, en contraste con las interacciones sociales simples que se basan en un solo tipo de confianza, los SACs suponen un equilibrio inestable entre estas tres dimensiones de la confianza.

La traducción y la comunicación ${ }^{13}$. La importancia de la "traducción" y los "traductores" en sistemas asociativos complejos puede remitirse a diversas líneas de reflexión sobre las redes como entidades complejas, que de manera más general han destacado la centralidad de las interfaces en las redes de redes, y la importancia de las actividades y el personal de frontera (los celadores, los analistas simbólicos, los negociadores o propiamente los traductores). Desde esta perspectiva, los traductores pueden ser concebidos como personas que facilitan la comunicación y la comprensión entre personas de diferentes instituciones, organizaciones o grupos que -como dirían Steward y Conway 1996- presentan desfases de lenguaje y orientación cognitiva.

La traducción está relacionada con la función de crear un lenguaje común a partir de diferentes orientaciones cognitivas y lógicas organizativas, y de re-significar la búsqueda de diferentes propósitos, preferencias, necesidades, intereses y causas hacia un objetivo común. Todo ello, sin vulnerar la autonomía política de la red y la autonomía individual de los participantes.

La traducción puede ser analizada tanto en el nivel estructural como en el individual. Al reunir a personas y entidades diversas, los SACs funcionan como estructuras de traducción. Estos sistemas conectan entidades y personas que provienen de diferentes contextos institucionales, organizativos y sociales, pero al mismo tiempo, dentro de los SACs, ciertos individuos cumplen el papel de traductores, facilitando la comunicación y la comprensión entre los miembros.

Por lo común, los traductores son personas que se han desempeñado en diversos tipos de organizaciones y entienden sus diferentes culturas y procedimientos; se relacionan con los otros participantes a través de una diversidad de vínculos formales e informales; poseen atributos personales que los hacen agradables y accesibles para los demás miembros, lo cual les permite actuar como facilitadores de la comunicación interpersonal; pueden ocupar posiciones centrales o marginales

\footnotetext{
${ }^{13}$ Para una discusión más amplia de este tema véase y el reporte de un estudio empírico sobre la traducción y los traductores véase Luna y Velasco (2003).
} 
REDES- Revista hispana para el análisis de redes sociales

Vol.17,\#4, Diciembre 2009

http://revista-redes.rediris.es

en la organización (Luna y Velasco 2003), y despliegan una intensa actividad de interacción en las áreas de toma de decisiones y resolución de problemas (Bonaccorsi y Piccaluga 1994:240).

En suma, la traducción básicamente responde a desfases en el lenguaje y orientaciones cognitivas, en los intereses y las lógicas organizacionales. Crea un campo común entre diferentes orientaciones cognitivas; reconcilia diferentes estructuras, mecanismos y procedimientos organizacionales; contribuye al desarrollo de un enfoque orientado a la solución de problemas; combina el conocimiento "local" y el "universal" y hace compatibles los diversos códigos que utilizan los participantes; y establece un lenguaje común para el procesamiento de luchas por el poder, a menudo relacionadas con las asimetrías y la variedad de recursos que se intercambian y redistribuyen en el sistema.

Dentro de ciertos límites parece haber una relación inversamente proporcional entre la confianza y la traducción. Esto se debe a que la importancia de los traductores depende del nivel de dificultad o facilidad de la comunicación entre los miembros, lo que a su vez depende de la confianza (Luna y Velasco, 2003).

Negociación y deliberación. Por su carácter, los SACs requieren de decisiones o acuerdos colectivos a los que se llega por medio de la negociación y el convencimiento.

Según Elster (1999), hay tres mecanismos básicos para tomar decisiones colectivas: el voto, la negociación o el regateo y la deliberación. Aunque no son mutuamente excluyentes, los mecanismos tienen diferencias fundamentales en sus presupuestos teóricos y normativos, y en sus posibilidades y limitaciones. El voto ha sido considerado un medio muy efectivo y eficiente para tomar decisiones, dado que generalmente produce resultados claros y rápidos (Jachtenfuchs 2007). Sin embargo, rara vez responde a los requisitos de los sistemas complejos que se caracterizan por la búsqueda del consenso -es decir, acuerdos sobre los cuales no hay oposición expresa de ningún participante- por medio de una mezcla de deliberación y negociación. Por lo tanto, nos concentraremos en las características, posibilidades y limitaciones de estos dos mecanismos para la toma de decisiones.

Negociación. La distinción central entre negociación y deliberación puede observarse desde el punto de vista de los intereses. Mientras que en la negociación los intereses son fijos y predeterminados, la deliberación supone la definición colectiva de las preferencias. La deliberación implica que los intereses no son externos al proceso político, sino que el debate y el intercambio de argumentos 
REDES- Revista hispana para el análisis de redes sociales

Vol.17,\#4, Diciembre 2009

http://revista-redes.rediris.es

transforman las preferencias, haciéndolas más compatibles. ${ }^{14}$

Una situación de negociación tiene varias características distintivas: hay dos o más participantes (individuales o colectivos); tienen conflictos de intereses que deben ser atendidos porque los participantes son interdependientes (es decir, se necesitan mutuamente); los involucrados quieren buscar un compromiso en lugar de pelearse abiertamente o salirse de la relación; y no hay reglas que automáticamente resuelvan el conflicto de intereses (Lewicki et al, 2004).

Los términos clave en estas definiciones son: interés, estrategia, conflicto y compromiso. Todos ellos aluden a formas de interacción cuyos participantes están simultáneamente en una relación de independencia y en competencia mutua. Esta competencia tiene por objeto determinar la forma en que se distribuyen los costos y beneficios de la interacción.

La negociación está anclada en varias características de los SACs, pero especialmente en la dependencia mutua de sus miembros. Mientras los participantes sean diversos y autónomos y cada uno de ellos, por su propia cuenta, controle recursos importantes y participe en la distribución del poder, la negociación siempre será necesaria. Esto la convierte en una característica esencial y permanente de los procesos de toma de decisión en sistemas complejos.

Pero otras características estructurales de los SACs apuntan hacia una dirección diferente. La negociación es posible sólo si los participantes tienen intereses claros que pueden ser especificados en objetivos y metas bien definidos; si esos objetivos pueden ser alcanzados mediante estrategias coherentes, orientadas hacia la obtención de beneficios concretos y hacia la minimización de pérdidas definidas de forma razonablemente precisa. Los participantes en SACs poseen varias de estas características, pero tienen otras muy diferentes o incluso opuestas.

En primer lugar, los intereses $-\mathrm{y}$ por lo tanto los objetivos, las metas, las estrategias, las ganancias y las pérdidas- no están por lo regular definidos de antemano, sino que se definen y redefinen dentro de la propia interacción. Es decir, son internos al sistema asociativo, son modificados e incluso generados dentro de él. Los problemas que las asociaciones normalmente tratan de resolver son complejos. Si los participantes (individuos u organizaciones) fueran capaces de definirlos de una forma que sea técnicamente correcta y a la vez ventajosa para ellos, realmente tendrían muy poca necesidad de sumarse a una asociación de este

${ }^{14}$ Sobre esta discusión, véase por ejemplo Magnette (2003), Eberlein y Kerwer (2002) y Smismans (2000). 
REDES- Revista hispana para el análisis de redes sociales

Vol.17,\#4, Diciembre 2009

http://revista-redes.rediris.es

tipo. La redefinición interactiva de los intereses y los problemas transforma fundamentalmente la interacción que se da dentro del sistema, distinguiéndolo claramente del regateo del mercado y de la negociación política. Antes de ser sujetos a negociación, estos intereses tienen que ser definidos mediante otros mecanismos de comunicación y toma de decisiones.

Pero, en segundo lugar, los SACs son más que simples mecanismos de intercambio. Son organizaciones autónomas, es decir actores colectivos propiamente dichos, con sus intereses, metas, estrategias, beneficios, pérdidas y problemas a resolver. Como se dijo antes, la confianza y la traducción tienen un papel crucial en la creación de un campo compartido y en la solidificación de la estructura de todo el sistema, aun cuando los participantes individuales continúan siendo autónomos e independientes.

La negociación está incrustada en esta estructura colectiva y por lo tanto ocupa un lugar más pequeño que el que tiene en el mercado y en las negociaciones políticas. Pero, puesto que muchas de las decisiones que se toman en los SACs se refieren no a la mejor manera de sacar provecho para satisfacer los intereses individuales, sino a la mejor solución a problemas comunes, estas decisiones deben ser acordadas mediante la argumentación más que negociadas.

Deliberación. En esencia, la deliberación se refiere al intercambio racional de argumentos para llegar a decisiones y soluciones razonables. Su meta principal es identificar un bien común, lo cual supone una redefinición de los intereses particulares.

Pero incluso cuando no logra identificar un bien colectivo, la evaluación deliberativa de distintos argumentos puede resultar por lo menos en una "evaluación colectiva de las divergencias" (Oléron, 1983: 108). Esta evaluación puede facilitar la mutua comprensión entre los miembros de la asociación. Pero igualmente importante es que esto inevitablemente lleva a los participantes a redefinir sus intereses, objetivos y metas. Como lo han mostrado varios autores, el proceso deliberativo compele a los participantes a presentar sus argumentos apelando a los intereses y valores comunes. Los intereses individuales se vuelven legítimos y públicamente defendibles sólo en la medida en que se puede argumentar que son compatibles, o por lo menos no son contradictorios, con los intereses comunes de los participantes. Esto también es especialmente importante en los SACs, porque los problemas que la interacción debe resolver tienen que ser definidos colectivamente o por lo menos deben ser redefinidos significativamente dentro de la propia interacción. 
REDES- Revista hispana para el análisis de redes sociales

Vol.17,\#4, Diciembre 2009

http: //revista-redes.rediris.es

En contraste con las soluciones de compromiso (y también con la coerción, la manipulación, la aceptación pasiva, la obediencia mecánica o las decisiones de mercado), la deliberación supone la justificación (Warren 1996). La deliberación se relaciona con capacidades y condiciones (tales como la autonomía en un doble sentido) que son necesarias para formular juicios colectivos de carácter discrecional. Los juicios son discrecionales en el sentido de que son formulados de forma libre y prudencial a través de debates guiados por la razón y el sentido común y que no son parciales o inapropiados.

Varias propiedades y características de los SACs facilitan la deliberación; entre ellas están la autonomía y la interdependencia de sus miembros y sus estructuras de poder descentralizadas. Pero incluso dentro de esta estructura favorable, la deliberación necesita condiciones óptimas. Entre las condiciones institucionales que pueden facilitarla pueden mencionarse la interacción regular y periódica entre un conjunto relativamente estable de participantes, que propicie el reconocimiento de los "otros", de sus percepciones, preocupaciones y preferencias; que genere confianza entre los miembros; que favorezca el aprendizaje político, y que propicie un compromiso con el mismo proceso deliberativo. Debe también asegurarse la comunicación y el flujo de información entre los participantes con el fin de crear las condiciones y generar los recursos de conocimiento e información para el ejercicio de una racionalidad argumentativa.

Comparada con otros métodos para tomar decisiones colectivas, la deliberación tiene varias ventajas distintivas. En contraste con el voto, donde la sujeción de la minoría a la decisión mayoritaria es un problema estructural, la negociación y la deliberación pueden producir resultados en los que no hay claros perdedores. Pero mientras que la negociación requiere idealmente el consenso de todos los actores estratégicos y por lo tanto la disposición de éstos a sacrificar algo para que sea posible lograr un acuerdo, la deliberación es un proceso para llegar a acuerdos cognitivos y normativos entre los participantes exclusivamente mediante la convicción mutua (ver Jachtenfuchs 2007). En otras palabras, mientras que el objetivo principal de la negociación es lograr un compromiso entre intereses en conflicto, la principal meta de la deliberación es convencer a los otros participantes. Así, los acuerdos colectivos logrados mediante la deliberación son cumplidos por la propia presión del "grupo de pares" y, por lo tanto, son menos vulnerables a la acción unilateral, que es una debilidad de los acuerdos negociados. 
REDES- Revista hispana para el análisis de redes sociales

Vol.17,\#4, Diciembre 2009

http: //revista-redes.rediris.es

La deliberación puede reforzar la eficacia de los SACs. De acuerdo con Weale (2001: 170), en ciertas circunstancias los procesos transparentes basados en la racionalidad deliberativa deben conducir a soluciones que son funcionalmente eficaces en la mayoría de los casos. Esto ocurriría si la solución a un problema determinado cumple con las siguientes condiciones: debe poder argumentarse que pertenece al conjunto de las decisiones que pueden ser elegidas razonablemente, incluso si hubo otras opciones que también pudieron haber sido elegidas razonablemente; debe estar abierta al escrutinio de quienes se benefician de la decisión y de quienes resultan perjudicados. Si esto es así, entonces la negociación y la urgencia por lograr la unanimidad se vuelven irrelevantes en la medida en que su resultado potencial pertenezca al conjunto de decisiones que pueden ser tomadas mediante la deliberación.

No obstante, la deliberación también tiene debilidades importantes. Muchas veces los acuerdos tienen un costo alto, puesto que normalmente son alcanzados mediante largos y complicados procesos de discusión. Este costo se incrementa porque siempre existe el riesgo de que al final de esos procesos no se pueda tomar una decisión (Jachtenfuchs 2007). La deliberación no sólo es un proceso que consume mucho tiempo; también requiere energía, atención, información y conocimiento, que han sido considerados recursos deliberativos escasos (Warren, 1996). Esto tiene consecuencias obvias para la eficiencia de los SACs.

En suma, las organizaciones que atribuyen un alto valor a la autonomía no pueden ser obligadas a ceder sus identidades y características constitutivas; sin embargo, el consenso facilita que la organización retenga a sus miembros, y a los miembros les permite preservar su propia autonomía e identidad (Brunsson y Olsen 1998 y Ahrne y Brunsson 2005: 442). De aquí la importancia de la negociación y la deliberación.

\section{Conclusión}

Debido a sus características peculiares, los sistemas asociativos complejos implican un equilibrio inestable entre las tres dimensiones de la confianza: normativa, estratégica y técnica; entre la negociación y la deliberación; en términos más generales, entre la integración y la autonomía (tanto individual como de la propia red).

El funcionamiento apropiado de las redes como sistemas asociativos complejos requiere la concurrencia de cuatro mecanismos de integración: la traducción, la confianza, la negociación y la deliberación. Sin embargo, dentro de ciertos límites, 
REDES- Revista hispana para el análisis de redes sociales

Vol.17,\#4, Diciembre 2009

http://revista-redes.rediris.es

hay una relación inversa entre dos pares de ellos: entre la confianza y la traducción, y entre la deliberación y la negociación. Cuando hay un nivel óptimo y una combinación adecuada de las tres dimensiones de la confianza, hay menor necesidad de traducción; el ejercicio exitoso de la deliberación hace que la negociación sea más fácil y menos importante como mecanismo para tomar decisiones

Lo anterior nos lleva a esperar que un SAC que funcione satisfactoriamente exhiba las siguientes características: una confianza fuerte y bien equilibrada que facilite la comunicación entre los participantes, una necesidad moderada de traducción, una práctica intensa de la deliberación para solucionar los conflictos internos y tomar decisiones colectivas, y un uso moderado de la negociación.

\section{Bibliografía}

Ahrne, Göran y Nils Brunsson (2005). "Organizations and Meta-organizations", Scandinavian Journal of Management, 21, pp. 429-449.

Bonaccorsi, A. y A. Piccaluga (1994). "A theoretical framework for the evaluation on university-industry relationships". R\&D Management, Manchester, Vol. 3, No. 4, pp. 229-247.

Brinton, H. Milward y J örg Raab (2002). “Dark Networks: The Structure, Operation and Performance of International Drug, Terror, and Arms Trafficking Networks". Ponencia presentada en Barcelona Workshop on Networks, Management and New Paterns of Governance. Barcelona, 4-5th October.

Brunsson, Nills y Johan P. Olsen (1998). “Organization Theory: Thirty Years of Dismantling, and then...?" En N. Brunsson y J. P. Olsen (eds), Organizing Organizations. Bergen: Fagbokforlaget, pp. 13-43.

Burt, Ronald S. (1992). Structural Holes. Cambridge, Mass: Harvard University Press.

Comisión Europea (2001). "Networking People for a Good Governance in Europe", Report of Working Group, White Paper on Governance, Work Area 4, Coherence and Cooperation in a Networked Europe (mayo). Texto elaborado por De Bruïne, Frans (pilot) y Giorgio Clarotti (rapporteur).

Eberlein, Burkard y Dieter Kerwer (2002). "Theorising the New Modes of European Union Governance." European Integration on Line Papers (EloP) 6, n. 5. Available online at http://eiop.or.at/eiop/texte/2002-005a.htm 
REDES- Revista hispana para el análisis de redes sociales

Vol.17,\#4, Diciembre 2009

http: //revista-redes.rediris.es

Elster, J on (1998). "A plea for mechanisms", en P. Hedström y r. Swedberg (eds.) Social mechanisms: An Analytical Approach to Social Theory. Cambridge: Cambridge University Press, pp. 45-73.

Elster, J on (1999). "The Market and the Forum: Three Varieties of Political Theory". En J. Bohman y W. Rehg (eds.), Deliberative democracy: essays on reason and politics. Cambridge, MA: MIT Press, pp. 3-34.

Granovetter, Mark. S. (1973). "The Strength of Weak Ties." American Journal of Sociology 78, n. 6, pp. 1360-80.

Hage, Jerald y Catherine Alter. (1997). “A Typology of Interorganisational Relations and Networks." En J. R. Hollinsworth y R. Boyer (eds.) Contemporary Capitalism. The Embeddedness of Institutions. Cambridge: Cambridge University Press, pp. 94126.

Hooghe, Liesbet y Gary Marks (2001). "Types of Multi-Level Governance." European Integration on Line Papers (EIOP) 5, n. 11.

Jachtenfuchs, Markus (2007). "The EU as a Polity (II)". En K. E. Jorgensen, M. Pollack y B. J. Rosamond (eds.) Handbook of European Union Politics, London: Sage, pp. 159-179.

Kanter, Rosabeth Moss y Derick Brinkerhoff (1981). “Organizational Performance: Recent Developments in Measurement". Annual Review of Sociology, Vol 7, pp. 321-349.

Lane, Christine (1998). "Introduction: Theories and Issues in the Study of Trust". En C. Lane and R. Bachman (eds.), Trust within and between Organizations: Conceptual Issues and Empirical Applications. Oxford: Oxford University Press, pp. $1-30$.

Lewicki, Roy J., et al. (2004). Essentials of Negotiation. Boston: McGraw-Hill/Irwin. Luna, Matilde (2008). “Las organizaciones colegiadas y las redes de gobernanza: entre la negociación y la deliberación". En C. Puga y M. Luna (eds.), Acción colectiva y organización. Estudios sobre desempeño asociativo. México: IIS-UNAM, pp. 343-405.

Luna, Matilde (2005). “Las redes de acción pública: ¿Un nuevo 'circuito' de la ciudadanía?" En B. Arditi(editor), ¿Democracia post-liberal?: El espacio político de las asociaciones, Editorial Anthropos/ Fac. Ciencias Políticas y Sociales, UNAM, Barcelona, Serie Argumentos de la Política: Pensamiento Crítico/ Pensamiento Utópico, Núm. 147, pp. 105-140. 
REDES- Revista hispana para el análisis de redes sociales

Vol.17,\#4, Diciembre 2009

http: // revista-redes.rediris.es

Luna, Matilde y José Luis Velasco (en prensa). “Mecanismos de toma de decisiones y desempeño en sistemas asociativos complejos", en M. Luna y C. Puga, Nuevas perspectivas en el estudio de las asociaciones, Barcelona: Anthropos/ IISUNAM.

Luna, Matilde y José Luis Velasco (2006). “Redes de conocimiento: principios de coordinación y mecanismos de integración". En M. Albornoz y C. Alfaraz, eds., Redes de conocimiento: construcción, dinámica y gestión. Buenos Aires: UNESCO, pp. 13-38.

Luna, Matilde y José Luis Velasco (2005). “Confianza y desempeño en las redes sociales." Revista Mexicana de Sociología 67, n. 1, pp. 127-162.

Luna, Matilde and José Luis Velasco (2003). "Bridging the Gap between Business Firms and Academic Institutions: The Role of Translators." Industry and Higher Education 17, n. 5, pp. 313-23.

Magnette, Paul (2003). "In the Name of Simplification. Constitutional Rhetoric in the Convention on the Future of Europe." Working Paper. Bruselas: Institut d'études européennes, Université Libre de Bruxelles.

March, James G. (1997). "Understanding how decisions happen in organizations". En Zur Shapira (ed.), Organizational decision-making. Cambrdige: Cambridge University Press, pp. 9-32.

Messner, Dirk (1999). “Del estado céntrico a la sociedad de redes. Nuevas exigencias a la coordinación social." En N. Lechner, R. Millán, and F. Valdés (eds.), Reforma del estado y coordinación social. Mexico: Plaza y Valdés, pp. 77-121.

Oléron, Pierre (1983). L'argumentation. Paris: PUF.

Sable, Charles (1993). "Studied Trust: Building New Forms of Cooperation in a Volatile Economy". Human Relations 46, no. 9: 1133-70.

Schmitter, Philippe (2001). "What is there to Legitimize in The European Union... and How Might this Be Accomplished?" Instituto Universitario Europeo, Florencia, Enero 2001.

Schneider, Volker, Achim Lang y Johannes M. Bauer (2006). “The adaptation of complex associational systems: Coordination and lobbying strategies of business associations in the context of globalization, and Europeanization". Ponencia presentada en la 64a . Conferencia Anual de la Midwest Political Science Association, Chicago, 20-23 de abril.

Smismans, Stijn (2000). "The European Economic and Social Committee: Towards Deliberative Democracy Via a Functional Assembly." European Integration on Line 
REDES- Revista hispana para el análisis de redes sociales

Vol.17,\#4, Diciembre 2009

http://revista-redes.rediris.es

Papers (EloP) 4, n. 12. Disponible en línea: http://eiop.or.at/eiop/texte/2000012a.htm

Steward, Fred y Steve Conway (1996). “Networks, Tacit Knowledge and Innovation." En R. Coombs, et al (eds.), Technological Collaboration: The Dynamics of Cooperation in Industrial Innovation. Cheltenham: E. Elgar, pp. 201-221.

Valente, Thomas (1995). Network Models of the Diffusion of Innovation. Cresskill, N.J.: Hampton Press.

Walker, Gordon, Bruce Kogut, y Wei-Jian Shan (1997). "Social Capital, Structural Holes and the Formation of an Industry Network." Organization Science 8, n. 2, pp. 109-25.

Warren, Mark E. (1996). “Deliberative Democracy and Authority." American Political Science Review 90, n.1, pp. 46-60.

Warren, Mark E. (2001). Democracy and Association. Princeton y Oxford: Princeton University Press.

Weale, Albert (2000). "Government by Committee. Three Principles of Evaluation." En T. Christiansen and E. Kirchner (eds.), Committee Governance in the European Union. Manchester: Manchester University Press, pp. 161-170. 